\title{
Non-invasive Intrauterine Administration of Botulinum Toxin A Enhances Endometrial Angiogenesis and Improves the Rates of Embryo Implantation
}

\author{
Hwa Seon Koo ${ }^{1} \cdot$ Min-Ji Yoon ${ }^{2} \cdot$ Seon-Hwa Hong ${ }^{1}$ • Jungho Ahn ${ }^{3} \cdot$ Hwijae Cha $^{2}$ - Danbi Lee ${ }^{2}$. Chan Woo Park ${ }^{4}$ \\ Youn-Jung Kang ${ }^{1,2,3}$ (D)
}

Received: 28 October 2020 / Accepted: 8 February 2021 / Published online: 1 March 2021

(C) The Author(s) 2021

\begin{abstract}
Endometrial angiogenesis plays crucial roles in determining the endometrial receptivity. Defects in endometrial receptivity often cause repeated implantation failure, which is one of the major unmet needs for infertility and contributes a major barrier to the assisted reproductive technology. Despite the numerous extensive research work, there are currently no effective evidence-based treatments to prevent or cure this condition. As a non-invasive treatment strategy, botulinum toxin A (BoTA) was administered into one side of mouse uterine horns, and saline was infused into the other side of horns for the control. Impact of BoTA was assessed in the endometrium at 3 or 8 days after infusion. We demonstrated that BoTA administration enhances the capacity of endothelial cell tube formation and sprouting. The intrauterine BoTA administration significantly induced endometrial angiogenesis displaying increased numbers of vessel formation and expression levels of related marker genes. Moreover, BoTA intrauterine application promoted the endometrial receptivity, and the rates of embryo implantation were improved with BoTA treatment with no morphologically retarded embryos. Intrauterine BoTA treatment has a beneficial effect on vascular reconstruction of functional endometrium prior to embryo implantation by increasing endometrial blood flow near the uterine cavity suggesting BoTA treatment as a potential therapeutic strategy for patients who are suffering from repeated implantation failure with the problems with endometrial receptivity.
\end{abstract}

Keywords Endometrial receptivity $\cdot$ Embryo implantation $\cdot$ Botulinum toxin A $\cdot$ Non-invasive treatment $\cdot$ Angiogenesis

\section{Introduction}

Angiogenesis is the process of new capillary formation from existing vascular structure by elongation or sprouting of endothelial cells, and is tightly regulated since most organs in the

Youn-Jung Kang

yjkang@cha.ac.kr

1 CHA Fertility Center Bundang, 59, Yatap-ro, Bundang-gu, Seongnam-si, Gyeonggi-do, South Korea

2 Department of Biomedical Science, School of Life Science, CHA University, 335 Pangyo, Bundang-gu, Seongnam-si, Gyeonggi-do, South Korea

3 Department of Biochemistry, Research Institute for Basic Medical Science, School of Medicine, CHA University, 335 Pangyo, Bundang-gu, Seongnam-si, Gyeonggi-do, South Korea

4 CHA Fertility Center Gangnam, 566, Nonhyeon-ro, Gangnam-gu, Seoul, South Korea adult usually do not undergo physiological angiogenesis unless they are not injured by trauma or infection [1-3], while the female reproductive tract requires continuous and cyclic angiogenesis for the growth of primordial follicles during the ovulation, receptive endometrium for the embryo implantation, and placental and embryonic development for successful pregnancy [4-6]. In particular, endometrial angiogenesis is a fundamental process for the menstrual cycle, endometrial receptivity, and embryo implantation [7]. Prior to embryo implantation, the endometrium undergoes dynamic changes of shedding and repair induced by ovarian steroid hormones to produce a period of uterine receptivity referred to as the "window of implantation" [8-10]. During the menstrual cycle, endometrial vasculature experiences continuous growth and regression, and coordinated vascular development induced by endometrial angiogenesis is essentially required for successful embryo implantation [11]. Direct or indirect association between implantation success and the endometrial angiogenesis facilitated by the 
conformational changes of end arterioles arising from arcuate arteries still need to be elucidated. Recent studies showing the increased expression levels of angiogenesis-related factor, VEGF, and its receptor in the late secretory and early pregnancy strongly suggest that the endometrial angiogenesis plays crucial roles in endometrial response to the implanting blastocyst, likely a key factor of endometrial vasculature in determining the endometrial receptivity $[3,12]$. Even though there are several trials to improve endometrial environment via inducing angiogenic effect with many kinds of substances including granulocyte colony-stimulating factor (G-CSF), mesenchymal stem cells (MSC), and platelet-rich plasma (PRP) [13-15], the efficacy of those substances is still controversial due to the lack of evidences $[16,17]$.

Botulinum toxin A (BoTA) is widely used in the plastic, reconstructive, and aesthetic surgery fields $[18,19]$. In addition to these usages, BoTA is commonly used in medical applications to treat chronic myofascial pain, headache, urinary incontinence, and hyperhidrosis [20-23]. Lately, there have been reports demonstrating the effect of BoTA on flap survival, which was suggested to occur by increased vasodilation and angiogenesis via elevated hypoxia-inducible factor (HIF) $1 \alpha$ and vascular endothelial growth factor (VEGF) [24, 25]. Moreover, BoTA is reported to enhance reepithelialization of human keratinocytes and angiogenesis of endothelial cells [26]. However, there was no report that evaluates the therapeutic effect of BoTA in the endometrium, especially endometrial angiogenesis, regarding the reproductive functionality. This led us to explore the impact of BoTA on endometrial angiogenesis and further investigation into the capacity of BoTA for the rates of embryo implantation for successful pregnancy via elevation of endometrial receptivity.

\section{Materials and Methods}

\section{Cell Culture and Botulinum Toxin A Treatment}

Human umbilical vein cells (HUVEC, ATCC) and human uterine microvascular endothelial cells (HUtMEC, PromoCell) were maintained in $\mathrm{EGM}^{\mathrm{TM}}-2$ endometrial cell growth medium 2 (Lonza) and endothelial cell growth medium MV (PromoCell), respectively. Ishikawa (ATCC) and CRL-4003 cells (kindly gifted from the laboratory of Dr. Haeng Seok Song) were maintained in DMEM/F12 media (Gibco, Grand Island, NY, USA) supplemented with $10 \%$ fetal bovine serum (Gibco, Grand Island, NY, USA) and 1\% penicillin-streptomycin (Gibco, Grand Island, NY, USA) as previously described [27]. All experiments were conducted using cells of the passages between 1 and 10. For the analyses for the effects of BoTA, cells were treated with commercially available BoTA (Botulax, Hugel, Seoul, Korea) for 24h, 48h, or $72 \mathrm{~h}$ at concentration of $0.5,2.0,10$, or 20IU. Botulax is the type for Clostridium botulinum toxin A derived from the strain of Clostridium Botulinum CBFC26, and for the utilization of BoTA from Botulax (Hugel) to perform the all analyses, the powder form of BoTA was dissolved and diluted in sterile $0.9 \%$ saline solution.

\section{Tube Formation Assays}

Assessment of the rates of endothelial tube formation in the presence or absence of BoTA (0, 0.5 IU, and 2.0 IU) was performed in a 96 -well plate. $2 \times 10^{4}$ HUVECs or HUtMECs were seeded onto growth factor reduced Matrigel matrix (\#354230, Corning Inc., Corning, NY, USA) pre-coated wells in 100ul of $\mathrm{EGM}^{\mathrm{TM}}-2$ endometrial cell growth medium 2 and endothelial cell growth medium MV, respectively. Following incubation at $37^{\circ} \mathrm{C}$ overnight, each well was analyzed directly under a microscope. The images $(\times 10$ magnification) were subsequently analyzed using ImageJ.

\section{Sprouting Assays}

Microfluidic device with the center channel of $800 \mu \mathrm{m}$ of width was used for HUVEC sprouting assays that were previously described [28]. The microfluidic chips were fabricated by polydimethylsiloxane (PDMS, Sylgard 184, Dow Corning) mold embedded with channel structures that was patterned by standard photolithography photoresist SU-8 (MicroChem). Demolded PDMS was punched out by using a biopsy punch $(6 \mathrm{~mm})$ and sharpened syringe needle $(0.5 \mathrm{~mm})$ to make reservoirs for the medium and hydrogel injection ports. A PDMS device and a glass coverslip were treated with oxygen plasma for $1 \mathrm{~min}$ before bringing them into contact. The devices were incubated in an $80^{\circ} \mathrm{C}$ dry oven for at least $48 \mathrm{~h}$ to restore hydrophobicity of PDMS. The devices were sterilized by UV irradiation before use. CRL4003 $\left(8 \times 10^{6} \%\right.$ $\mathrm{ml})$ cells were mixed with fibrinogen solution $(2.5 \mathrm{mg} / \mathrm{ml}$ fibrinogen with $0.15 \mathrm{U} / \mathrm{ml}$ aprotinin). Thrombin $(0.5 \mathrm{U} / \mathrm{ml})$ was added to cell mixture and immediately introduced into the channel for stromal cell. Fibrinogen solution $(2.5 \mathrm{mg} / \mathrm{ml}$ of fibrinogen with $0.15 \mathrm{U} / \mathrm{ml}$ of aprotinin) mixed with thrombin $(0.5 \mathrm{U} / \mathrm{ml})$ was applied to the vessel channel. After $3 \mathrm{~min}$, the upper reservoirs of each device were filled with EGM-2 culture medium and aspirated gently from the lower reservoirs to wet the hydrophobic media channel. Subsequently, HUVECs $\left(5 \times 10^{6} / \mathrm{ml}\right)$ were plated into the media channel. The device was then tilted $90^{\circ}$ in an incubator for $40 \mathrm{~min}$ to attach the cell mixture to the gel-media interface. The device with multilayer of cells was incubated for 7 days until fully lumenized microvessels had formed. A schematic illustration of cell loading and generation of perfused microvessel on the device is shown in Fig. 1. To quantify the area for angiogenic sprouting of HUVECs, cells were immunostained against CD31 (abcam; ab28364, 1:100) and detected its expressed 
Fig. 1 The impact of BoTA treatment on the capacity of tube formation and sprouting of endothelial cells. Representative three images showing tube formation of HUVECs $(\mathbf{a}-\mathbf{b})$ and HUtMECs (c-d) in the condition of BoTA $(0,0.5,2.0 \mathrm{IU})$ treatment for $48 \mathrm{~h}$. Scale bar: $200 \mathrm{um}$. The total number of tube loops was quantified in graphs shown in (b) and (d). (e) A schematic diagram of a micro-engineered vascular system that was used for the analyses of angiogenic effect of BoTA. CRL4003 stromal cells were mixed in fibrin matrix and then plated in the stromal cell channel. HUVECs attached on the wall of fibrin gel were patterned in the central channel. Scale bar: $800 \mu \mathrm{m}$. (f)

Representative confocal microscopic images of angiogenic sprouting of HUVECs in response to BoTA. Scale bar: 100 $\mu \mathrm{m}$. The total surface area of HUVEC sprouting was quantified in a graph shown in (g). Data shown in (b), (d), and (g) are from 3 independent experiments and analyzed using the ordinary oneway ANOVA analysis with

Dunnett's multiple comparison test including $P$-values $(*<0.05$, $* *<0.01, * * *<0.001$,

$* * * *<0.0001, N S$ not significant)

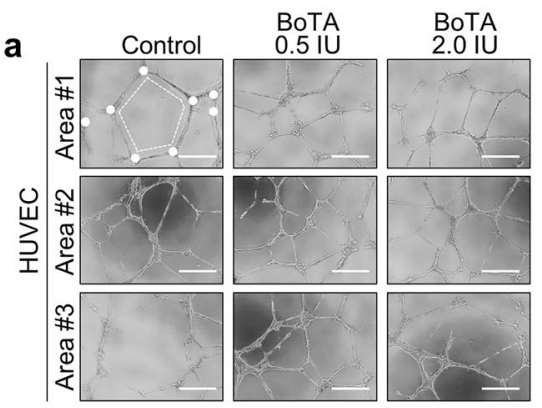

b

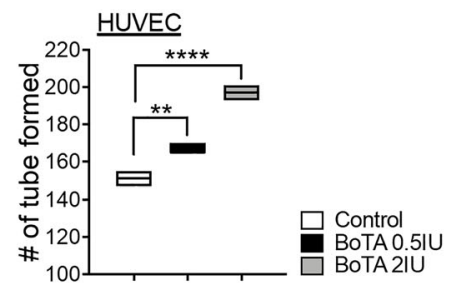

e

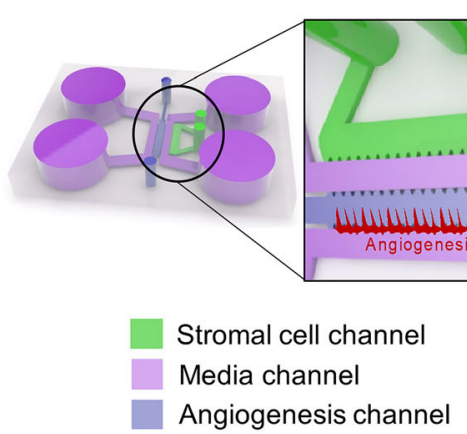

f
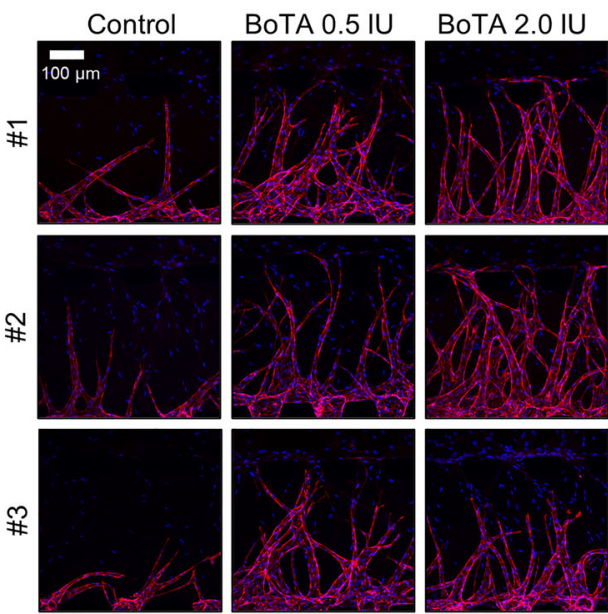

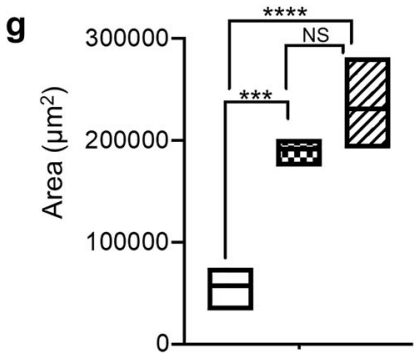

Control

B BoTA-0.5IU

BoTA-2.0IU area using confocal microscope. Z-projections of the 3D stacks of microvascular networks were obtained each day with IMARIS (Bitplane) and further analyzed with ImageJ (National Institutes of Health, Bethesda, MD) to obtain the binary images. The proportion of fluorescent pixels within the region of interests of each image was calculated.

\section{Quantitative RT-PCR-Based Analysis of mRNA Expression}

SYBR Green (Roche, Basel, Switzerland) assays were used to quantitate angiogenesis- and embryo implantation-related genes in BoTA-treated and control samples. Total RNA extracted using TRIzol reagent (Ambion, Life Technologies Corporation, CA, USA) at 1 ug was converted to cDNA using M-MLV reverse transcriptase (Promega, Madison, WI, USA), dNTP (Invitrogen, Carlsbad, CA, USA), and oligo dT primer (Labopass, Seoul, Korea). The efficiency of the primer sequences was tested by creating an external standard curve. Synthesized cDNA was sequentially diluted with the dilution factor 1:10 four times $(1 / 1,1 / 10,1 / 100$, and 1/1000). Amplifications were run where each primer pair was loaded into wells containing different cDNA concentrations in a CFX Connect ${ }^{\mathrm{TM}}$ Real-Time PCR Detection System (BioRad, 
Hercules, CA, USA). A standard curve was generated for each primer by plotting the log value of the starting quantity of template against the cycle threshold value obtained during the amplification of each dilution displaying $R^{2}$ value greater than 0.98 and an amplification efficiency around $100 \%$. With $1 / 10$ volume of cDNA, gene expression was quantitatively analyzed. A DNA melting-curve was used to confirm the presence of a single PCR product in each assay. Real-time PCR results for angiogenesis- and embryo implantationrelated genes, which were measured by $\mathrm{C}_{\mathrm{T}}$ values, were normalized to $C_{T}$ values of $\beta$-actin and evaluated as a percentage of expression levels measured in cells of control group (the ratio for interesting gene/ $\beta$-actin is quantified by $2^{\mathrm{CT}(i n t e r e s t i n g}$ gene)-CT( $\beta$-actin) $)$. All values were analyzed using the ordinary one-way ANOVA analysis with Dunnett's multiple comparison tests. Primer sequence pairs used for these analyses are shown in Supplementary Table 1.

\section{Immunofluorescence and Microscopy}

Immunofluorescence staining was performed as previously described [29]. Cells were fixed with $4 \%$ of paraformaldehyde and were specified and permeabilized for $15 \mathrm{~min}$ using $0.05 \%$ Triton X-100/PBS. Subsequently, cells were blocked using $5 \% \mathrm{w} / \mathrm{v}$ bovine serum albumin (BSA) in PBS for $1 \mathrm{~h}$ at room temperature. Localization studies were performed using primary antibody to HIF1- $\alpha$ (Cell signaling technology; \#3434, 1:50), integrin $\alpha \mathrm{v} \beta 3$ (MILLIPORE; MAB1976, 1:100), LIF (NovusBiologicals; NBP1-85717, 1:100), CD31 (abcam; ab28364, 1:100), and CD34 (abcam; ab8536, 1:100) and further incubated with anti-rabbit IgG fluorescence (Invitrogen) or anti-mouse IgG fluorescence (Invitrogen) as appropriate, all at 1:400 for $1 \mathrm{~h}$. Cover glasses were mounted in Vectashield mountant with 4',6-diamidino-2-phenylindole (DAPI; Vector Laboratories) as nuclear stain. For the negative control, cells were incubated under similar conditions with isotype-matched mouse or rabbit immunoglobulin in the place of the primary antibodies. Images were captured using oil immersion 63x objectives Zeiss 510 microscopy (Carl Zeiss MicroImaging, Röttingen, Germany) and processed using Zen software (ZEISS).

\section{Immunohistochemistry}

Histology and immunohistochemistry were performed as previously described [27]. Tissues were fixed with $4 \%$ of paraformaldehyde and embedded in paraffin. Paraffin-embedded tissue sections $(5 \mu \mathrm{m})$ were deparaffinized in Histoclear (National diagnostics; HS-202) and dehydrated with gradient absolute ethanol (80-100\%) for $3 \mathrm{~min}$ each. Antigen retrieval was performed in sodium citrate buffer $(\mathrm{pH} 6.0)$ for $1 \mathrm{~h}$ at $95^{\circ} \mathrm{C}$. Endogenous peroxidase activity was blocked using $3 \% \mathrm{H}_{2} \mathrm{O}_{2} / \mathrm{PBS}$ for $10 \mathrm{~min}$ at room temperature and washed with PBS. Subsequently, non-specific binding sites were blocked with $1 \%$ BSA/PBS for $1 \mathrm{~h}$. Sections were incubated overnight at $4{ }^{\circ} \mathrm{C}$ with primary antibody to integrin $\beta 3$ (Cell Signaling; \#13166, 1:200) and OPN (Enzo; ADI-905-629, 1:100). After three washes with PBS, sections were incubated with biotinylated goat anti-rabbit secondary antibodies (Vector laboratories; ZG0122 1:200) for $30 \mathrm{~min}$ at room temperature. The signal was detected using Avidin-Biotin HRP (Vector Laboratories; DK-6100, Vectastain Elite ABC Kit) with DAB substrate solution (Thermo; VE296420, DAB substrate kit). Sections were counterstained with hematoxylin (BioGNOST; HEMH-46/20), dehydrated in a graded ethanol for 3 min each, cleared in Histoclear, and mounted with Permount medium (Fisher chemical; 196934). For the negative control, cells were incubated under similar conditions with isotype-matched mouse or rabbit immunoglobulin in the place of the primary antibodies. Images were captured using Olympus CKX53 microscopy (Olympus Life Science) and processed using eXcope.

\section{Animal Uses and BoTA Infusion}

All experiments were conducted under a Home Office license and the Animal Act, 1986, and had local ethical approval for care and use of laboratory animals. C57BL/6 strain mice were maintained by strict accordance with the policies of the CHA University Institutional Animal Care and Use Committee (IACUC, approval number 190126). All mice were housed under standard environmental conditions of $12 \mathrm{~h}$ light: $12 \mathrm{~h}$ dark at a controlled room temperature $\left(20-22^{\circ} \mathrm{C}\right.$ and 40 $60 \%$ humidity) and fed ad libitum. Five-week-old female and 7 -week-old male mice provided by Orientbio (Gapyeong, Gyeonggi, South Korea) were used for the analyses and evaluation of impact of BoTA. The female mice were anesthetized via intraperitoneal injection of tribromoethanol (Avertin). A vertical incision was made to gently expose the uterus through the abdominal wall. BoTA was prepared in 30ul of saline and softly instilled into one side of mouse uterine cavities through the uterotubal junction using an insulin syringe equipped with a 31-gauge needle, and saline was infused into the other side of horns for the control. The overall operation time of a mouse was no more than $15 \mathrm{~min}$, and the unilateral uterine horn administration time was about $5 \mathrm{~s}$. After 8 days of BoTA infusion, female mice were weakly stimulated for the ovulation with $2.5 \mathrm{IU}$ of pregnant mare serum gonadotropin (PMSG, Daesung Microbiological, Korea), and ovulation was synchronized by $1.25 \mathrm{IU}$ of human chorionic gonadotropin (hCG, Sigma, USA) 46-48h later, both by intraperitoneal injection. Females were placed singly with the same strain male mice overnight. The presence of a vaginal plug the following morning (day 1 of pregnancy) was used as an indicator of successful mating. Both sides of uterine horns were obtained 16 days after mating for further analyses of 
pregnancy outcomes. Implantation sites and embryos were isolated, and the total numbers were counted to analyze the rates of embryo implantation. Isolated embryos were weighed individually, and their individual sizes and growth morphology were examined.

\section{Mouse Embryo Collection and Co-culture}

For the embryo collection, female mice (6-8 weeks) were superovulated with $10 \mathrm{IU}$ of PMSG, and ovulation was synchronized by $5 \mathrm{IU}$ hCG 46-48h later, both by intraperitoneal injection. Females were placed singly with males of the same strain overnight. The morning of the presence of a vaginal plug was designated day 1 of pregnancy. Pregnant mice were killed on day 1 . One-cell embryos were obtained from the oviduct using a $30 \mathrm{G}$ dissecting needle of $1 \mathrm{ml}$ syringe to tear open the ampulla of the oviduct and release. Cumulus cell around of the one-cell embryo were washed with $0.1 \%$ hyaluronidase drop at $37^{\circ} \mathrm{C}$ for $5-10 \mathrm{~min}$ and dissociated by gentle pipetting to glass pipettes. Collected embryos were washed with M2 media (Sigma, USA) supplemented with $4 \mathrm{mg} / \mathrm{ml}$ BSA washed in KSOM (Millipore, USA) and cultured in a 20ul drop of KSOM covered with mineral oil at $5 \% \mathrm{CO}_{2}$, $37^{\circ} \mathrm{C}$ until the blastocyst stage. Only expanded blastocysts with clearly observable inner cell mass and trophectoderm on day 5 were included in the study. Day 5 mouse embryos were transferred onto saline- or BoTA-treated Ishikawa cells in independent wells of 24-well plate. Multiple observations of stability for embryo attachment were performed to identify the distinct stages of attachment from 12 to $48 \mathrm{~h}$ of co-culture as previously reported $[27,29]$. Standardized plate movement protocol was applied to assess the stability of embryo attachment. The plate was tapped three times laterally and orthogonally to detect unattached embryos. Five stages of attachment were defined and used as a measurement scale as previously described [29, 30].

\section{Library Preparation and Sequencing for RNA-Seq Analysis}

For control and test RNAs, the construction of library was performed using QuantSeq 3' mRNA-Seq Library Prep Kit (Lexogen, Inc., Austria) according to the manufacturer's instructions. In brief, each 500ng total RNA was prepared, and an oligo-dT primer containing an Illumina-compatible sequence at its 5' end was hybridized to the RNA, and reverse transcription was performed. After degradation of the RNA template, second strand synthesis was initiated by a random primer containing an Illumina-compatible linker sequence at its 5' end. The double-stranded library was purified by using magnetic beads to remove all reaction components. The library was amplified to add the complete adapter sequences required for cluster generation. The finished library is purified from PCR components. High-throughput sequencing was performed as single-end 75 sequencing using NextSeq 500 (Illumina, Inc., USA). The raw and normalized data have been deposited in the Gene Expression Omnibus (GEO) database (accession number: GSE146934, Supplementary Table 2).

\section{Data Analysis}

QuantSeq 3' mRNA-Seq reads were aligned using Bowtie2 (Langmead and Salzberg, 2012). Bowtie2 indices were either generated from genome assembly sequence or the representative transcript sequences for aligning to the genome and transcriptome. The alignment file was used for assembling transcripts, estimating their abundances and detecting differential expression of genes. Differentially expressed genes were determined based on counts from unique and multiple alignments using coverage in Bedtools (Quinlan AR, 2010). The $\mathrm{RC}$ (read count) data were processed based on quantile normalization method using Edge R within $\mathrm{R}$ ( $\mathrm{R}$ development Core Team, 2016) using Bioconductor (Gentleman et al., 2004). Gene classification for gene ontology (GO) and pathway analysis was performed by DAVID (http://david.abcc. ncifcrf.gov/) and Medline databases (http://www.ncbi.nlm. nih.gov/). Classified genes and their fold enrichment values were visualized into dotplots by using $\mathrm{R}$ and automatically categorized by ClueGO [31, 32]. The significance cutoffs were set for fold change $(\geq 2.0), P$-value $(<0.05)$, and FDR $(<0.05)$.

\section{Cell Proliferation and Cytotoxicity Assay}

Cell proliferation assay of Ishikawa cells was performed by cell proliferation assay kit (BIOMAX, Seoul, Korea) according to the manufacturer's instructions. $1 \times 10^{4}$ cells were seeded and maintained in the presence or absence of BoTA at indicated concentrations for $24 \mathrm{~h}, 48 \mathrm{~h}$, or $72 \mathrm{~h}$. Absorbance was measured using microplate reader (Thermo Scientific, Rockford, IL, USA) at 450nm wavelength followed by adding 10ul QuantiMAX ${ }^{\mathrm{TM}}$ mixture (BIOMAX, Seoul, Korea). Cytotoxicity of Ishikawa cells depending on BoTA treatment was measured using EZ-LDH ${ }^{\mathrm{TM}}$ cytotoxicity assay kit (BIOMAX, Seoul, Korea) according to the manufacturer's instructions. $5 \times 10^{3}$ Ishikawa cells were treated with BoTA at indicated concentrations for $24 \mathrm{~h}, 48 \mathrm{~h}$, or $72 \mathrm{~h}$, and absorbance was measured by adding $10 \mathrm{ul}$ of $\mathrm{LDH}$ reaction mixture. Reaction of LDH mixture was performed on a shaker for $90 \mathrm{~min}$, and then the number of survived cells was counted.

\section{Wound Healing Assay}

Ishikawa cells were seeded in 6-well plate and cultured to $100 \%$ confluence. After $24 \mathrm{~h}$ of starvation, a linear scratch was generated using a sterile $1 \mathrm{ml}$ pipette tip. Cells were 
treated with BoTA at indicated concentrations for $48 \mathrm{~h}$. Images of wound closure were taken using inverted microscope (Olympus Corp., Tokyo, Japan), and the gap distances of wound closure were analyzed using ImageJ.

\section{Statistical Analysis}

Comparison groups were analyzed with unpaired Student $t$ test for parametric distributions. For multiple comparisons, the ordinary one-way ANOVA analysis with Dunnett's multiple comparison test was used. For all cases, a $P$-value that was $<0.05$ was considered statistically significant $\left(P<0.05\left(^{*}\right)\right.$, $P<0.01(* *), P<0.001(* * *)$, and $P<0.0001(* * * *)$ ).

\section{Results}

\section{BoTA-Induced Enhancement of Angiogenesis in Endothelial Cells In Vitro}

To investigate the effect of BoTA treatment on the tube formation ability of human endothelial cells, we plated HUVECs onto Matrigel-coated plate, and 0.5IU or 2.0IU of BoTA was applied. The tube formation as measured by the total number of tube loops (indicated with dash line in the image) and branching points (indicated with white dots in the image) was significantly higher in BoTA-treated HUVECs compared to saline-treated controls (Fig. 1a-b). More specifically, the assessment of tube formation of human uterine endothelial cells (HUtMEC) revealed that BoTA induced the angiogenic effect exhibiting dramatically higher numbers of tube formation in BoTA (0.5IU)-treated groups compared to control (Fig. 1c-d). Interestingly, unlike HUVECs, HUtMECs showed no further significant effect in tube formation at higher dose (2.0IU) of BoTA treatment. Furthermore, we implemented a micro-engineered 3-dimensional angiogenesis system to explore the effect of BoTA on the ability of vessel formation and sprouting using HUVECs and endometrial stromal CRL4003 cells. The device consists of 5 primary channels; 2 fluidic microchannels separate 3 hydrogel-laden microchannels from each other to facilitate the supply of fresh media through the device (Fig. 1e). This co-culture system enables paracrine interaction between HUVECs and endometrial stromal layer, mediating the angiogenic morphogenesis. The microengineered angiogenesis model was exposed to BoTA at $0.5 \mathrm{IU}$ or $2.0 \mathrm{IU}$. These analyses revealed that BoTA treatment induced an increasing effect on angiogenesis displaying promoted angiogenic sprouting of HUVECs in BOTA-treated group compared to control $(0.5 \mathrm{IU} ; P=0.0003,2.0 \mathrm{IU}$; $P<0.0001)$ (Fig. 1f), which is consistent with the data from tube formation assay. This increasing angiogenic effect was further quantified through the analysis of the sprout area (Fig. $1 \mathrm{~g})$.

\section{BoTA-Induced Elevation of Endometrial Receptivity Markers in Endometrial Cells In Vitro}

To investigate the effect of BoTA in the endometrial cells in regard to the regulation of endometrial receptivity, BoTA was applied to endometrial epithelial (Ishikawa) and stromal (CRL4003) cells in vitro. Quantitative RT-PCR analyses revealed that BoTA treatment induces significant increases of mRNA expression levels of endometrial receptivity-related genes including ITGB3, HIF1- $\alpha$, VEGFR2, VIMENTIN, IL-6, and IL-8 in both Ishikawa and CRL4003 cells (Fig. $2 \mathrm{a}-\mathrm{b})$ suggesting both endometrial epithelial and stromal cells are responsive to BoTA treatment. Particularly, our data show that endometrial epithelial cells are more sensitive to BoTA treatment rather than stromal cells, which are evidenced by the findings showing that Ishikawa cells were more responsive to BoTA with a short-term treatment compared to CRL4003 cells. Even though both Ishikawa and CRL4003 immortalized cells might lack normal physiological features compared to primary endometrial cells, these data might implicate that endometrial epithelial layer is the more effective target of BoTA to induce the faster response of endometrial receptivity. Induction of endometrial receptivity-related genes was validated with immunofluorescence (IF) staining analyses, displaying higher expression of HIF $1 \alpha$, integrin $\alpha \mathrm{v} \beta 3$, and LIF in BoTA-treated group compared to control (Fig. $2 \mathrm{c}$ and Supplementary Fig. 1a-b). Of note, relatively higher concentration (10IU) of BoTA treatment rather led to reduction of all the expressions of endometrial receptivity-related markers (Supplementary Fig. 1c-d). Furthermore, to evaluate the toxicity or safety of BoTA treatment, we performed the cellbased in vitro assays to test if BoTA treatment induces cytotoxicity or aberrant cell proliferation in endometrial epithelial cells prior to further investigation for the clinical applicability of BoTA treatment. These analyses revealed that BoTA treatment induces impact on neither cytotoxicity nor aberrant cell proliferation in endometrial epithelial cells regardless of the concentration ranges between $0.5 \mathrm{IU}$ and $20 \mathrm{IU}$ (Supplementary Fig. 2a-b). Additionally, BoTA-induced migratory effects of endometrial cells were assessed. Fully confluent cells in the condition of starvation were scratched, and their migratory capacity was assessed by measuring the gap distance in between of two sides of cells (Supplementary Fig. 2c-d). No significant differences were observed in BoTAtreated (0.5 and 2.0IU) groups compared to controls indicating BoTA has no stimulatory effect on migratory capacity of both endometrial epithelial and stromal cells (Supplementary Fig. 2e-f). Our observations here demonstrate that BoTA treatment at an appropriate concentration increases endometrial receptivity-related gene expression in both endometrial epithelial and stromal cells in vitro implicating that BoTA treatment might induce positive regulatory effect of endometrial receptivity. 

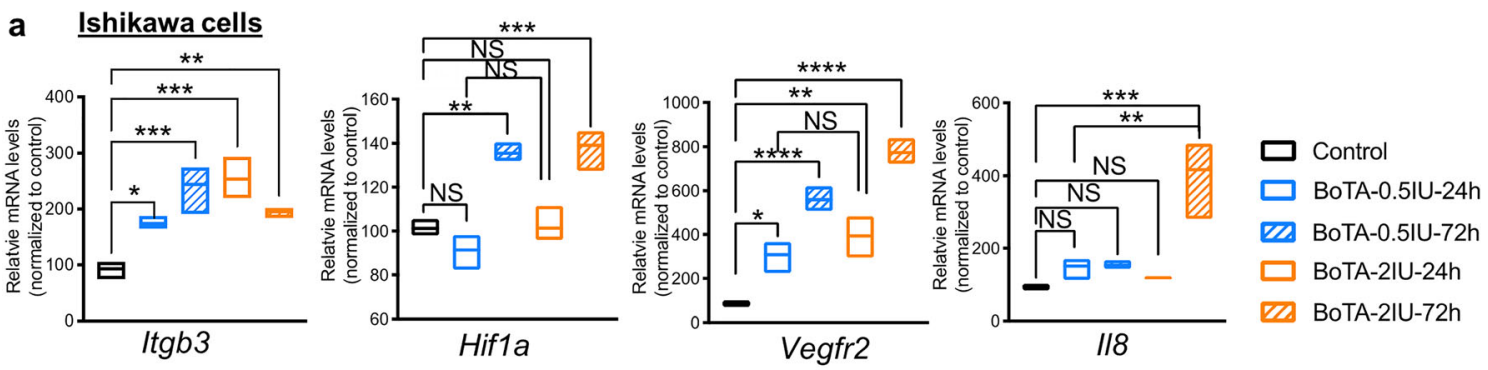

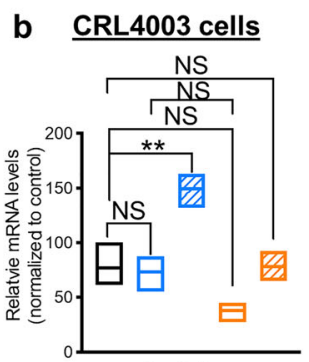

$\operatorname{ltg} 3$

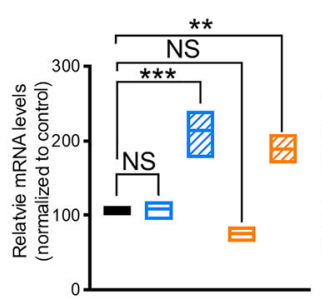

118

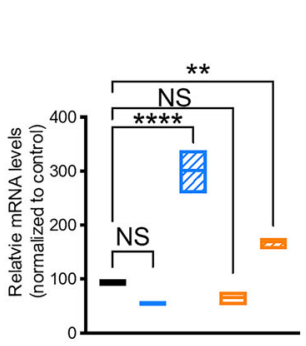

$1 / 6$

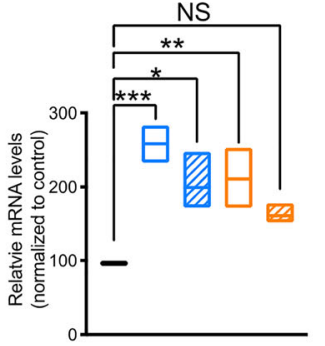

Vimentin

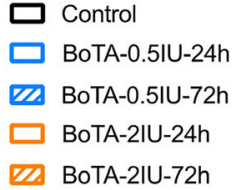

BoTA-2IU-72h

C
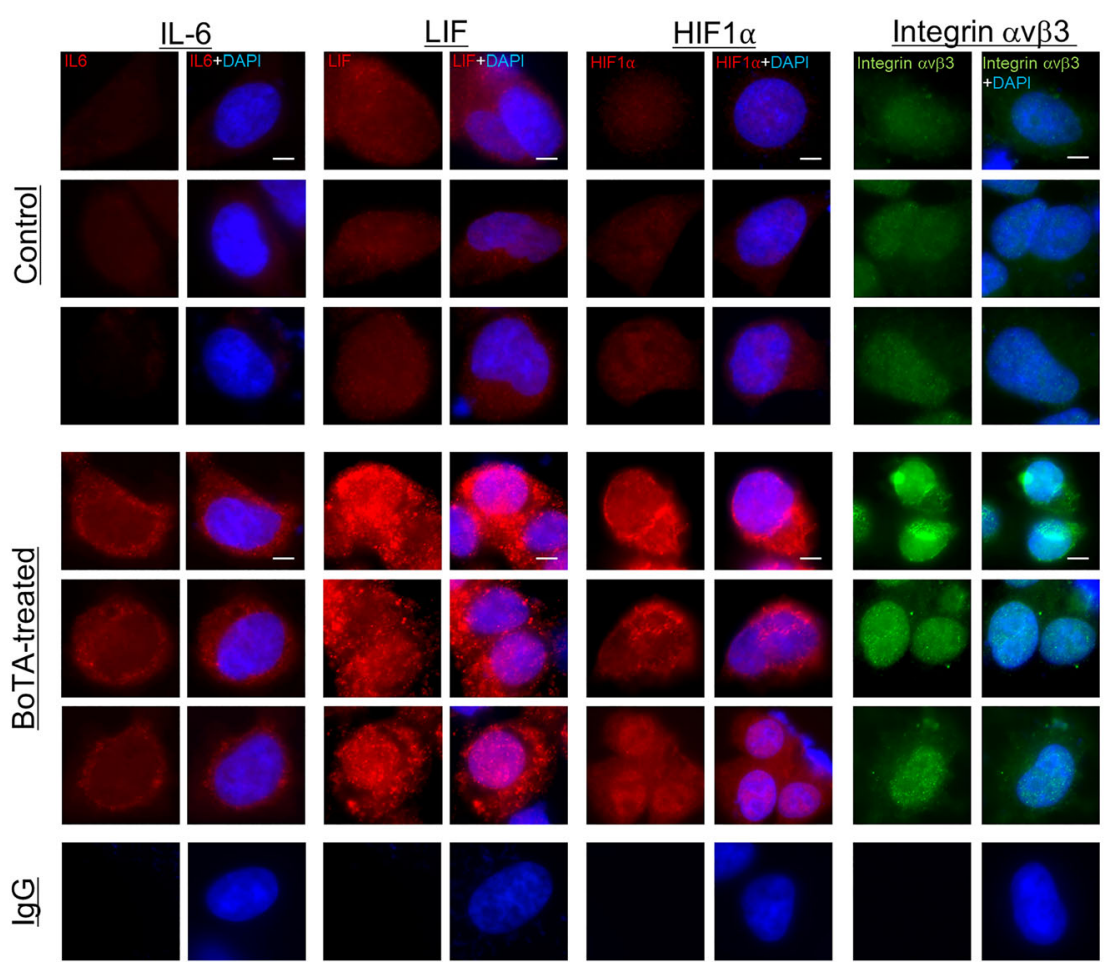

Fig. 2 Endometrial receptivity-related factors are induced by BoTA treatment in endometrial cells. QRT-PCR analyses of Itgb3, Hifla, Vegfr2, and $I l 8$ in endometrial epithelial (Ishikawa) cells (a), and Itgb3, Il8, Il6, and Vimentin in endometrial stromal (CRL4003) cells (b). (c)

\section{Identification of Alterations in Gene Expression Induced by Intrauterine BoTA Infusion in Mice}

To examine the in vivo effects of intrauterine application of BoTA, BoTA was infused into one side of mouse uterine horns, and saline was infused into the other side of horns for
Immunofluorescence (IF) staining of IL-6, LIF, HIF1- $\alpha$, and integrin $\alpha v \beta 3$ in Ishikawa cells in response to BoTA. Saline-treated cells were used for control. For the negative control, mouse or rabbit IgG was used. Scale bar: 20um

the control (Fig. 3a). Mouse uterine tissues were obtained 3 or 8 days after BoTA infusion, and total RNA was extracted from the whole endometrial tissues (Fig. 3b). In order to further examine the alterations of global gene expression depending on the intrauterine application of BoTA, RNA-seq data were generated from BoTA-treated versus saline-treated 
a

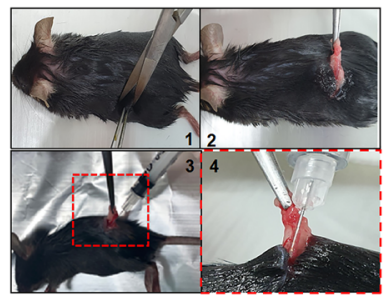

b
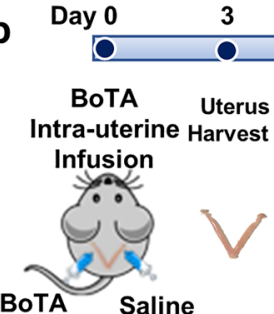

d

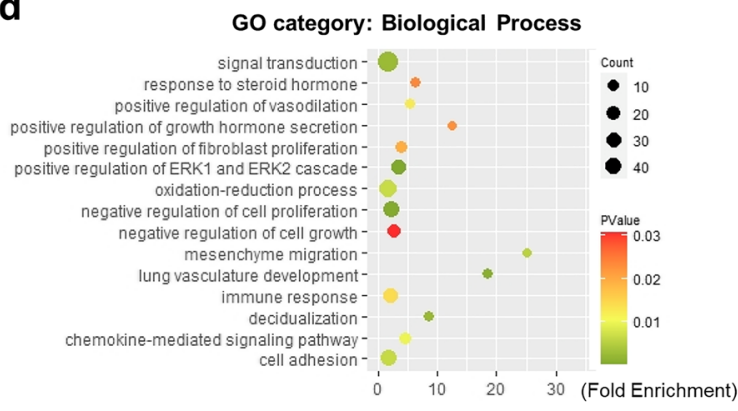

GO category: Cellular Component

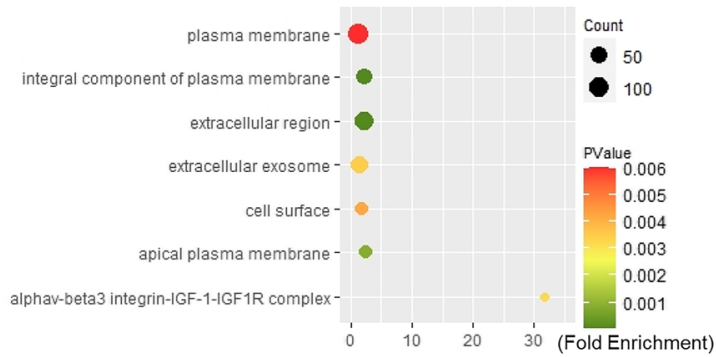

GO category: Molecular Function

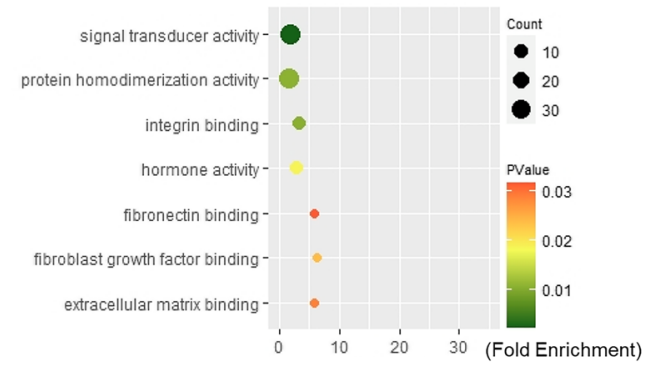

GO category: Kegg Pathway

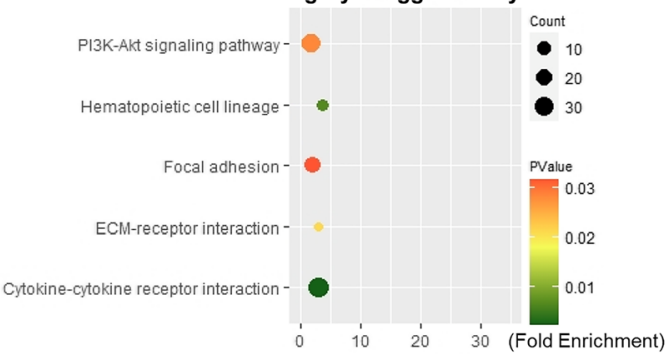

(control) endometrial tissues. Unsupervised hierarchical clustering analyses using a fold change cutoff of 2 and a $P$-value cutoff of 0.05 identified a total of 197 (88 genes were upregulated and 109 genes were down-regulated) and 573 (266 genes were up-regulated and 307 genes were down-regulated)
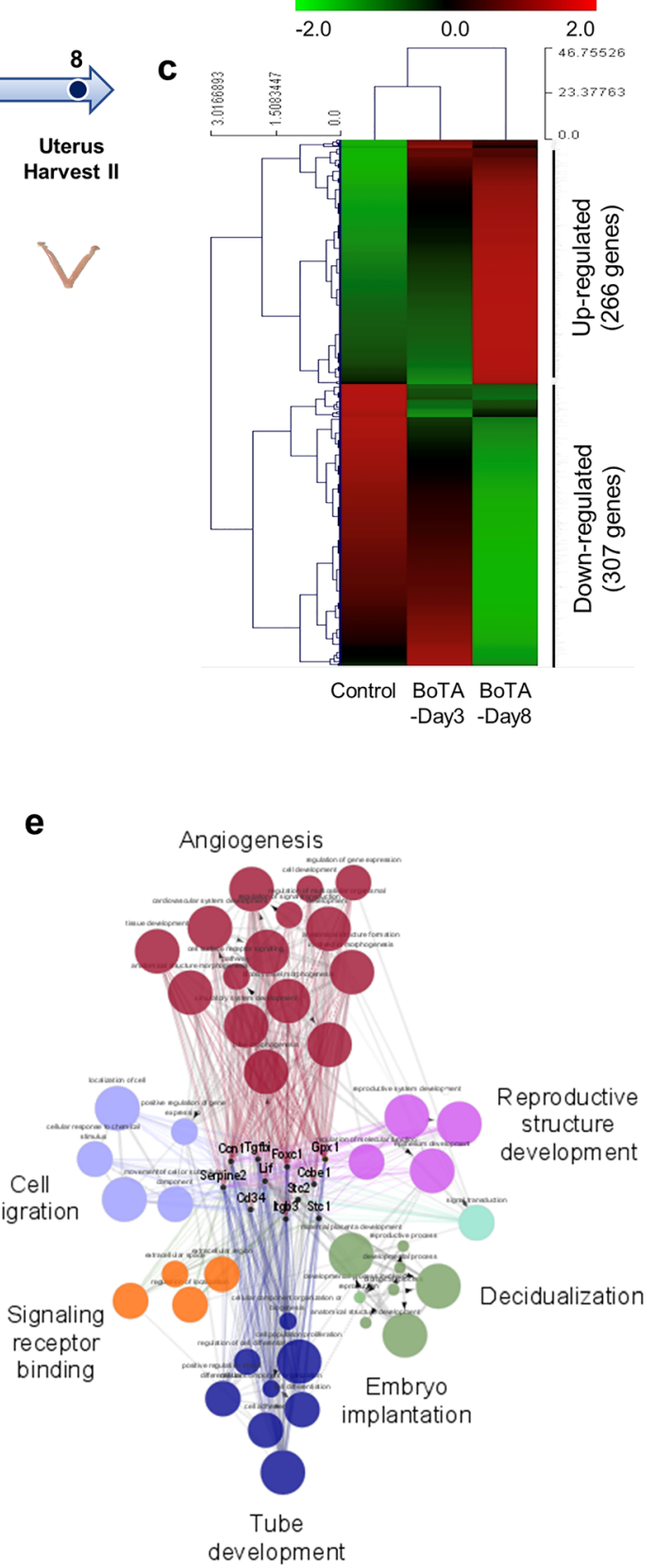

differentially expressed genes in BoTA-Day 3 and Day 8 groups, respectively (Fig. 3c and Supplementary Fig. 3a). To perform functional clustering of 573 differentially expressed genes, which were captured from Day 8 BoTA-treated uterine tissues, gene ontology (GO) and pathway analyses were 
Fig. 3 Identification of differentially expressed genes between the BoTA-treated uterus vs. control. (a) A procedure of intrauterine BoTA administration. (b) A schematic diagram of experimental plan of intrauterine BoTA administration. (c) Unsupervised hierarchical clustering analysis of RNA seq visualizing a heatmap plot showing differentially expressed genes (DEGs) in BoTA-treated group vs. control. Each row represents a distinct sample (5 independent BoTA-treated groups compared to saline-treated control), and each column represents an individual gene (a list of 23,282 genes). Normalized $(\log 2)$ and standardized (each sample to mean signal $=0$ and standard deviation $=1$ ) level of gene expression is denoted by color (green, low; dark, intermediate; red, high), as indicated in the gradient panel. (d) Dot plots displaying gene ontology (GO) and pathway analysis of differentially expressed genes between BoTA-treated (Day 8) and control using DAVID tool and their fold enrichment on $\mathrm{X}$-axis and gene count numbers on dot size. The cutoff for significance was set by $P<0.05$. Biological process (BP), cellular component (CC), and molecular function (MF), KEGG pathway (KP) annotations. (e) A network of gene-gene interaction among BoTA-regulated genes was constructed displaying specifically enriched signaling pathways

performed by using the Database for Annotation, Visualization, and Integrated Discovery (DAVID) online tools [33]. Enriched GO terms in each category and pathway including associated gene counts, $P$-value, and FDR are shown in Table 1 . The $P$-value and fold enrichment (FE)value were calculated by Fisher's exact test and multiple comparisons test, respectively $(P<0.05$ and FE $>1.5)$. A total of 573 differentially expressed genes were classified according to GO terms, including biological process, BP; cellular component, CC; molecular function, MF; and KEGG pathway, KP (Fig. 3d and Table 1). These analyses demonstrate that specific BP categories, including response to steroid hormone ( $P=0.023429$ ), positive regulation of growth hormone secretion $(P=0.022572)$, decidualization $(P=0.002337)$, positive regulation of vasodilation ( $P=0.012491)$, lung vasculature development $(P=0.001022)$, and cell adhesion $(P=0.006471)$, were enriched in BoTA-treated (Day 8) uterine tissues implicating biological processes particularly related to angiogenesis and the early phase of embryo-endometrial interaction in response to hormones. Moreover, enriched terms of the $\mathrm{CC}$ category include integral components of plasma membrane $\left(P=4.39 \mathrm{xE}^{-08}\right)$, apical plasma membrane $\left(P=8.52 \mathrm{xE}^{-04}\right)$, extracellular region $\left(P=6.03 \mathrm{xE}^{-14}\right)$, and cell surface $(P=0.004213)$. Among the enriched $\mathrm{CC}$ components, integrin $\alpha \mathrm{v} \beta 3$-integrin IGF1-IGF1R complex $(P=0.003231)$ is specifically reported to be strongly correlated to the early stage of embryo implantation [27]. Integrin binding ( $P=0.010497)$, signal transducer activity $(P=0.002136)$, hormone activity $(P=0.018643)$, fibroblast growth factor binding $(P=0.023895)$, and extracellular matrix binding activity $(P=0.029068)$ were composed of enriched MF terms. Pathway enrichment analyses based on KEGG pathway (KP) analyses were performed using either Chi-square test or Fisher's exact test, revealing that pathways including cytokine-cytokine interaction $\left(P=1.30 \mathrm{xE}^{-04}\right)$ and
PI3K-AKT signaling pathway ( $P=0.032839)$, hematopoietic cell lineage $(P=0.005628)$, focal adhesion $(P=0.037505)$, and ECM-receptor interaction $(P=0.023927)$ were enriched in BoTA-treated (Day 8) uterine tissues (Fig. 3d and Table 1). These analyses implicate that intrauterine BoTA treatment might induce the intracellular signaling including ERK and PI3K-AKT pathway to possibly result in promoting angiogenesis [34, 35], which is supported by enrichment of signal transducer activity classified in BP and MF categories. Moreover, intercellular interactions might be enhanced in BoTA-treated endometrium, which was evidenced by high levels of responses including cytokine-cytokine receptor interaction $\left(P=1.30 \mathrm{xE}^{-04}\right)$, chemokine-mediated signaling pathway $(P=0.009934)$, immune response $(P=0.014198)$, and oxidation-reduction process $(P=0.006635)$. Furthermore, indepth clustering analyses revealed that BoTA treatment are strongly associated with the regulation of decidualization, hormonal activity, and interaction between surface adhesion molecules and ECM, which are critically correlated with endometrial receptivity, the process of embryo implantation, and maintenance of pregnancy [36]. Further analyses of enriched GO categories of Day 8 BoTA-treated endometrium compared to saline-treated group, visualized by ClueGO, revealed that BoTA-induced enrichment includes angiogenesis-related pathways such as angiogenesis, cell migration, signaling receptor binding, reproductive structure development, tube development, embryo implantation, and decidualization (Fig. 3e). Among these enrichments, 13 significantly differentially expressed genes including Ccl7, Cyr61, Itgb3, Foxc1, Clec14a, Hif3a, Gpx1, Cd34, Ccbe1, Tgfbi, Lif, Stc1, and Stc2 were specifically associated with GO terms of angiogenesis (GO:0001525) or embryo implantation (GO:0007566) (Table 2).

\section{Enhanced Endometrial Blood Vessel Formation by Intrauterine BoTA Treatment}

In order to validate our RNA-seq data, genes that are classified and strongly related to angiogenesis including $\mathrm{Ccl7}$, Cry61, Itgb3, Clec14a, Hif3a, Gpx1, Cd34, Ccbe1, Tgfbi, Vegfr1, $V e g f a$, and Tiel were subjected to quantitative QRT-PCR analyses. The validation revealed that the expression pattern examined by QRT-PCR was concordant with RNA-seq data (Fig. 4a). To further corroborate the evidence for the induction of endometrial angiogenesis with BoTA infusion in mouse uterus, our interrogation of paraffin-embedded BoTA-treated endometrial tissue sections with IF staining of CD31, a surrogate marker for blood vessel formation, revealed that BoTA treatment increased CD31 expression in mouse uterus compared to control group (Fig. 4b, supplementary Fig. 4a-b), which is consistent with our RNA-seq analyses. In particular, BoTA-infused endometrial tissues exhibited enhanced CD31 levels in the stromal layer especially close to the lining of 


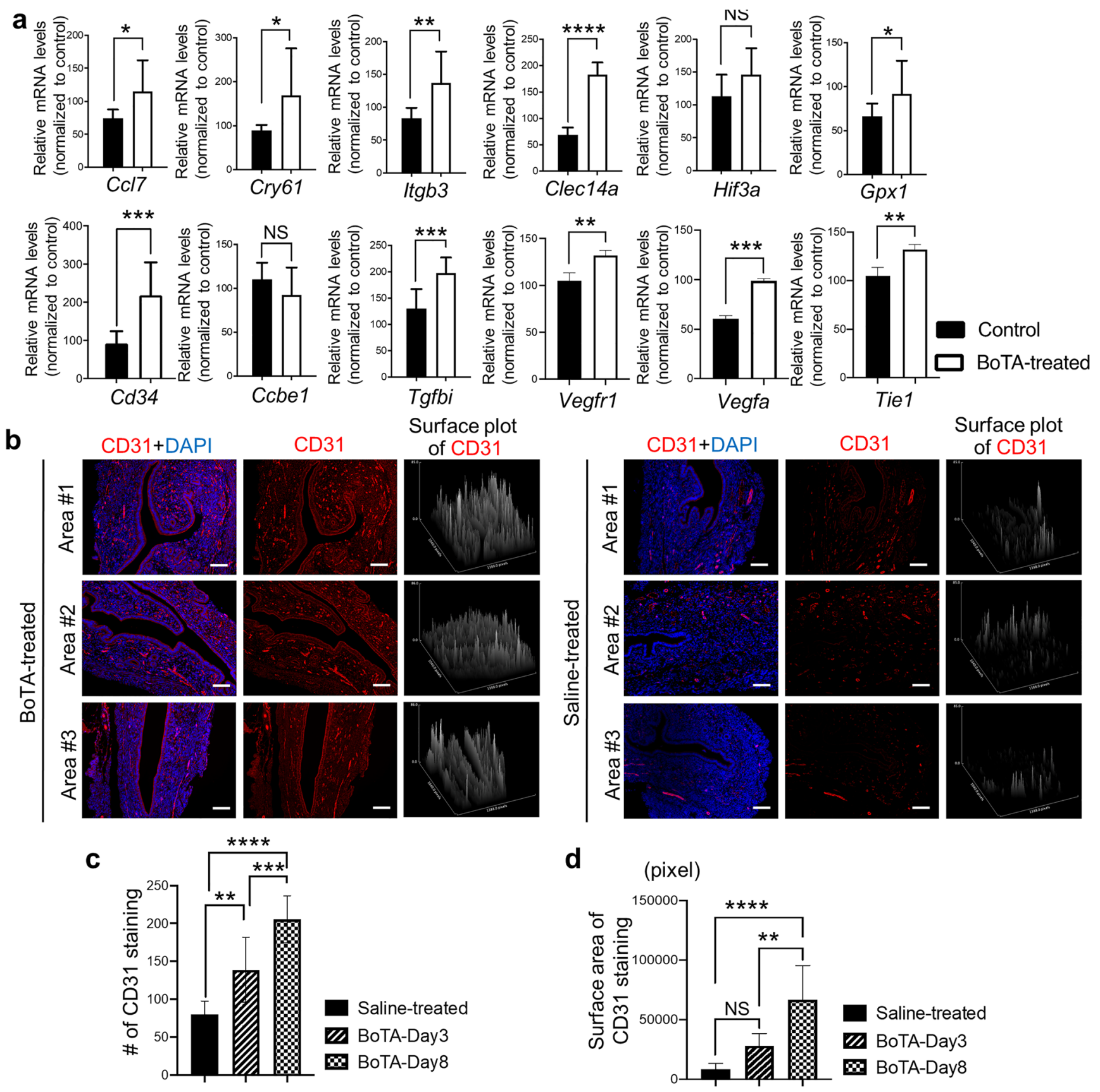

e

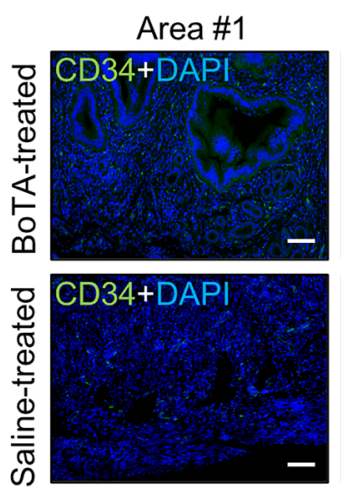

Area \#2

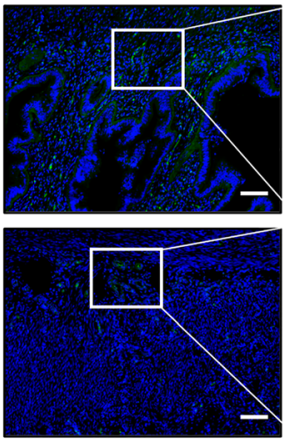

Magnified
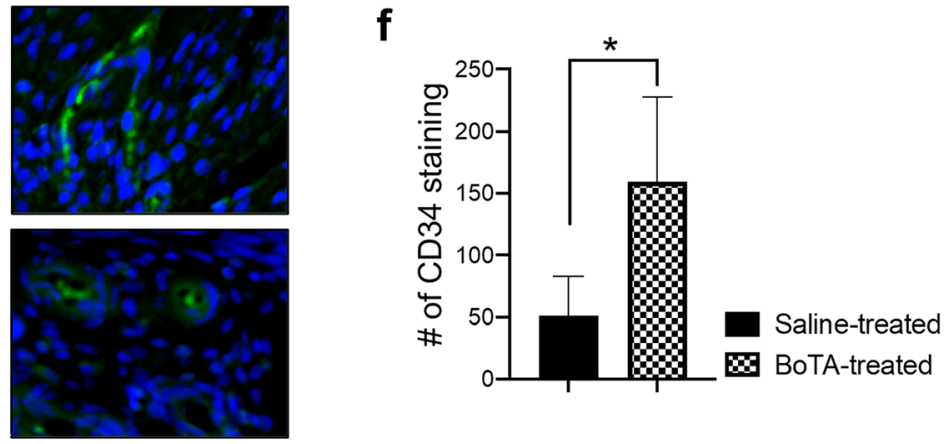
Fig. 4 Induction of angiogenesis-related genes in BoTA-administered mouse uterus. (a) QRT-PCR analysis of Ccl7, Cry61, Itgb3, Clec14a, Hif3a, Gpxl, Cd34, Ccbel, Tgfbi, Vegfrl, Vegfa, and Tiel in BoTAtreated uterus samples compared to controls. (b) IF staining of CD31 in longitudinally sectioned mouse uterus harvested 8 days after BoTA intrauterine infusion. Saline-treated endometrium was used for control. Scale bar: 100um. Representative two images from the different areas are shown in Supplementary Figure 4A. CD31 expression was quantified by counting the number of CD31 staining (c) and stained surface area (d). (e) IF staining of CD34 in longitudinally sectioned mouse uterus harvested 8 days after BoTA intrauterine infusion. Saline-treated endometrium was used for control. Scale bar: 100um. CD34 expression was quantified by counting the number of CD34 staining (f). Comparison groups for (a) and (f) are from 3 independent experiments and analyzed using the unpaired Student $t$-test for parametric distributions and the multiple comparisons for (c) and (d) are from 3 independent experiments and analyzed using the ordinary one-way ANOVA analysis with Dunnett's multiple comparison test including $P$-values $(*<0.05, * *<0.01$, $* * *<0.001, * * * *<0.0001, N S$ not significant)

epithelium. Moreover, the total number and surface area of CD31 staining were further quantified exhibiting significant and gradual increase in BoTA-treated groups (Day 3 and 8) compared to control (Fig. 4c-d). As a novel marker for angiogenesis of vascular endothelial cells and progenitor cells with stemness [37], CD34 expression pattern was assessed in paraffin-embedded BoTA-treated endometrial tissue sections revealing more abundantly expressed CD34 in BoTA-treated groups compared to control (Fig. 4e-f, Supplementary Fig. 4b). These observations indicate that BoTA infusion might have an angiogenic effect to enhance the development of new capillaries from pre-existing blood vessels in the endometrium.

\section{Improved Endometrial Receptivity by Intrauterine BoTA Treatment}

Among significantly altered embryo implantation-related genes, differential expression pattern of Itgb3 and Lif upon BoTA treatment showed consistency with data from in vitro analyses shown in Fig. 2. In order to further support our RNA-seq data, genes that are classified and strongly related to embryo implantation, including integrin $\beta 3$, osteopontin (OPN), LIF, STC1, and STC2, were subjected to immunohistochemistry or QRT-PCR analyses. Integrin $\beta 3$ and its ligand OPN proteins were remarkably highly expressed in BoTA-treated endometrial tissue section compared to saline-treated control group (Fig. 5a-b). The validation with QRT-PCR revealed concordant pattern of expression of Lif, Stc1, and Stc2 with those from RNAseq analyses (Fig. 5c-e), suggesting that intrauterine infusion of BoTA might be an effective method to improve the endometrial receptivity for patients who are suffering from implantation failure with poor receptivity.

\section{BoTA-Treated Uterine Fertility Assessment}

Angiogenesis is known to participate in a wide range of the process in pregnancy including folliculogenesis, early implantation, placentation, and embryonic development [2, 38]. This led us to examine the correlation of enhanced neovascular activity and increased molecular features of endometrial receptivity induced by BoTA treatment with the outcomes of embryo implantation and pregnancy. For the assessment of BoTA effect on the stability of embryo attachment in vitro, a total of 73 day 5 mouse embryos were transferred (one embryo per well) to confluent Ishikawa cells, which were treated with BoTA (primed; 5h of exposure to BoTA prior to embryo co-culture, non-primed; commence BoTA exposure at the time of embryo co-culture) and co-cultured for $19 \mathrm{~h}, 24 \mathrm{~h}$, $28 \mathrm{~h}$, or $45 \mathrm{~h}$. The stability of attached mouse embryo was assessed according to the 5-stage standard: score 1, floating; 2, weakly attached but detached after tapping; 3, weakly attached but stuck at the attachment site after tapping; 4, stably attached; and 5, stably attached and showed outgrowth [29, $30]$. Up to $24 \mathrm{~h}$ of co-culture, embryos transferred onto both control and BoTA-treated cells showed no significant difference in their attachment stability. However, at the late stage of co-culture (24-48h), embryos were more stably attached onto BoTA-primed treated Ishikawa cells compared to controls $(P=0.0332$ ) (Fig. 6a). Of particular note, no significant difference was observed in the stability of attached embryo onto non-primed BoTA-treated Ishikawa cells compared to that of control cells $(P=0.6322)$ (Supplementary Fig. 5a), suggesting an effective time-point for BoTA treatment to improve the rates of embryo implantation, which should be primed for the embryo transfer onto endometrial epithelial cells. In order to verify the efficacy of BoTA in vivo, BoTA was applied to mouse uterus in a same manner as shown in Fig. 3a. After 8 days of BoTA infusion, both sides of uterine horns were obtained to expose the embryo implantation sites 16 days after mating (Fig. 6b). These analyses revealed that the total number of implantation sites in BoTA-treated group was significantly higher than controls $(P=0.0121)$ (Fig. 6c-d). Intriguingly, increased numbers and thickness of uterine arteries, which formed the network towards each site of embryo implantation in mouse uterus, were observed at the open abdominal surgery in BoTA-administered side compared to control (Fig. 6e). This might be because of increased endometrial neomicrovessles, which might have been developed and stimulated from pre-existing uterine arteries, induced by BoTA intrauterine treatment. Importantly, no retarded embryos were detected in both groups, and the weight of embryos from both groups showed no significant difference (Fig. 6f-h). Representative images of the implantation sites in the uterus of mice and embryos obtained from different conditions are shown in Fig. 6c and $\mathrm{f}$. 
a

\begin{tabular}{c} 
Integrin $\beta 3$ \\
\hline Area \#1 $\quad$ Area \#2
\end{tabular}
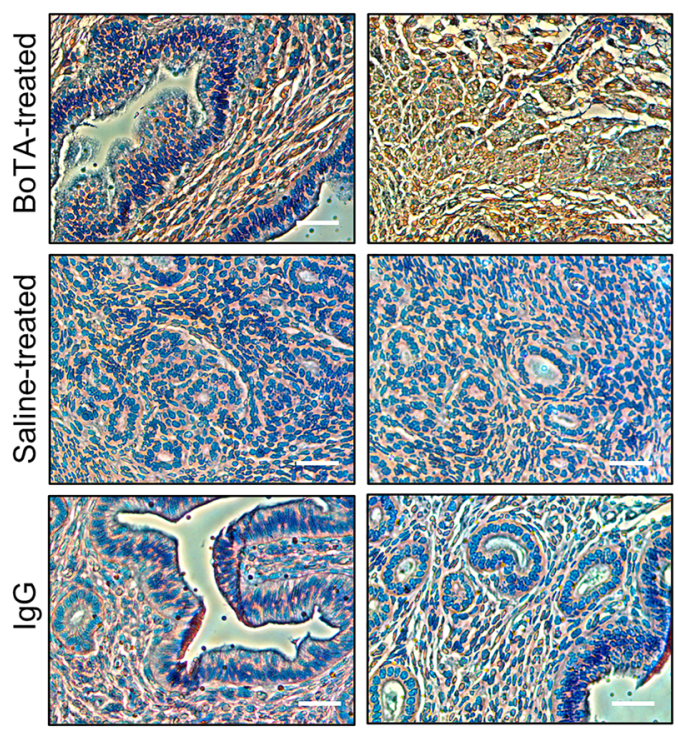

C

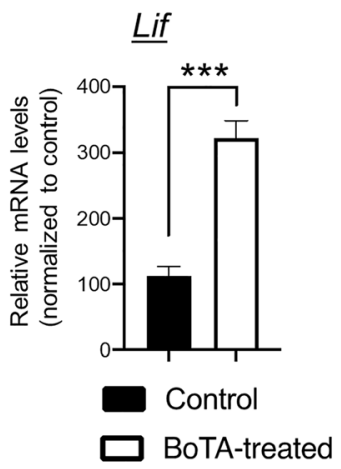

d

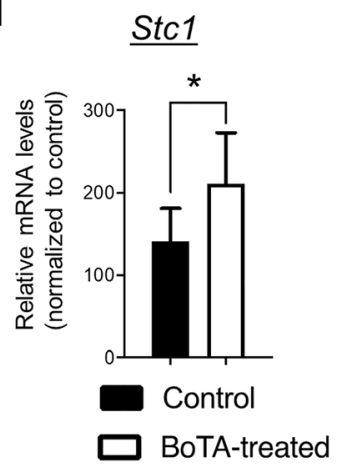

b

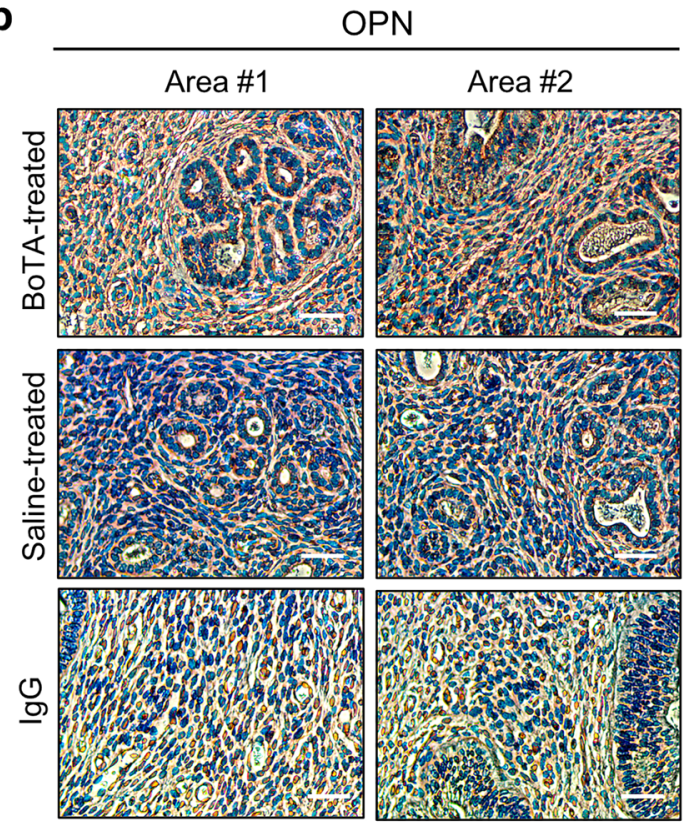

e

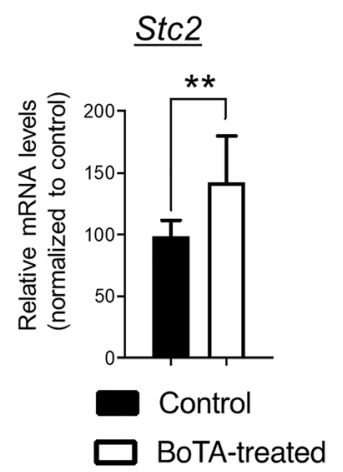

Fig. 5 Induction of endometrial receptivity-related genes in BoTAadministered mouse uterus. Representative two images of immunohistochemistry analyses of integrin $\beta 3$ (a) and OPN (b) in longitudinally sectioned mouse uterus harvested 8 days after BoTA intrauterine infusion. Saline-treated endometrium was used for control. For the negative control, mouse or rabbit IgG was used. Scale bar: 20um. QRT-PCR analysis of Lif (c), Stc1 (d), and Stc2 (e) in BoTA-treated uterus samples compared to controls. Comparison groups for $(\mathbf{c}-\mathbf{e})$ are from 3 independent experiments and analyzed using the unpaired Student $t$-test for parametric distributions including $P$-values $(*<0.05, * *<0.01$, ***<0.001)

\section{Discussion}

In this current study, we demonstrate that the intrauterine BoTA administration significantly induces endometrial angiogenesis displaying increased numbers of vessel formation, which is consistent with the findings of increased tube formation and sprouting area of endothelial cells in vitro. Furthermore, BoTA administration enhances the endometrial receptivity and subsequently improves the rates of embryo implantation with no morphologically retarded embryos. Even though the molecular and cellular mechanisms underlying BoTA-induced endometrial angiogenesis and its direct or indirect correlation with embryo implantation remains to be defined, this might suggest intrauterine application of BoTA, which is totally non-invasive causing no traumatic injury to the endometrium at all, as an effective therapeutic strategy for patients who are suffering from repeated implantation failure with the problems with endometrial receptivity.

Endometrial angiogenesis is thought to be fundamental in supporting endometrial growth and repair that provide vascularized endometrial receptivity, which usually refers to the ability of the endometrial lining to accept and accommodate a nascent embryo $[38,39]$. To support this notion, it has been previously reported that increased endometrial blood flow is associated with the pregnancy outcome during the time of window of implantation [40]. This can be evidenced by our observations displaying the enhanced capacity of endothelial cell tube formation and sprouting towards the endometrial stromal cell layer with BoTA treatment, implicating the impact of BoTA on the increased vascular network and blood 
a

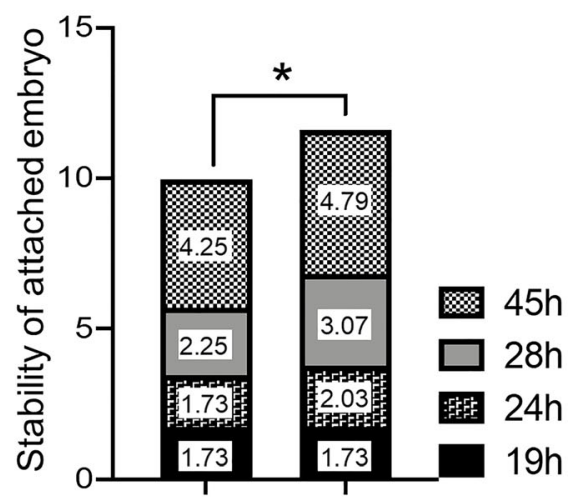

b

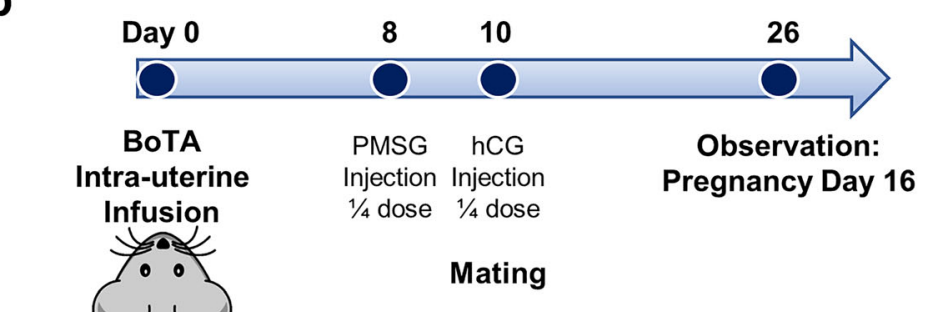

C
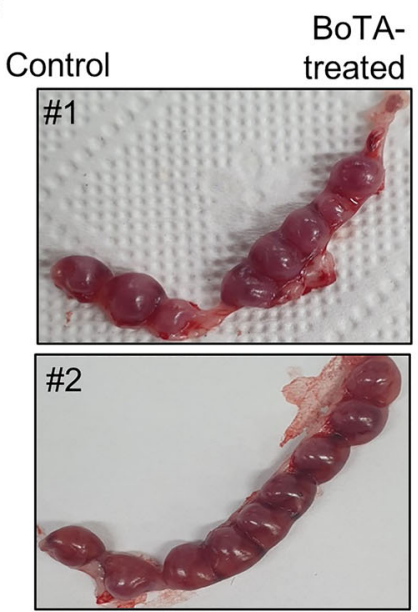

f

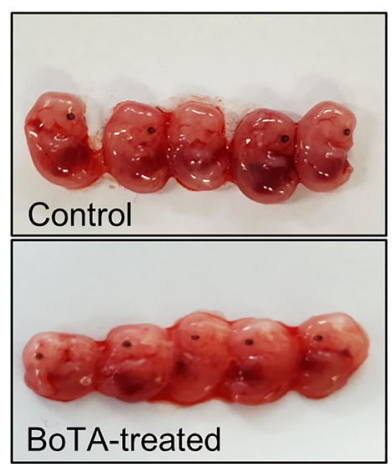

d

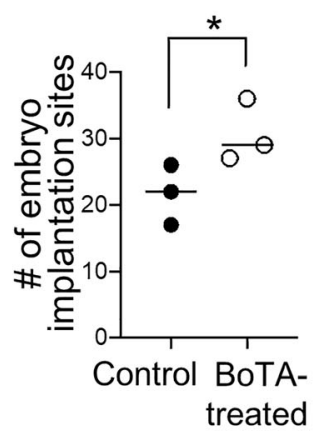

g

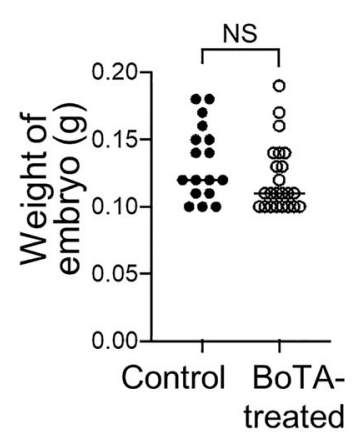

e

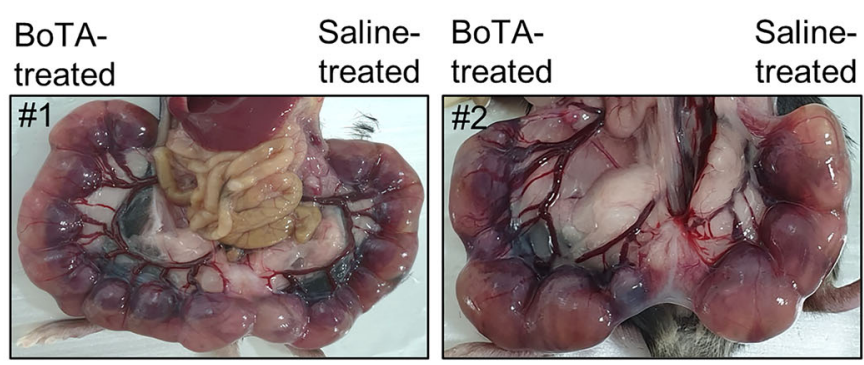

h

\begin{tabular}{|l|l|}
\hline & $\begin{array}{l}\text { Embryo weight } \\
(\mathrm{n}=157)\end{array}$ \\
\cline { 2 - 2 } & Average + SD \\
\hline Control & $0.14 \mathrm{~g} \pm 0.043$ \\
\hline $\begin{array}{l}\text { BoTA- } \\
\text { treated }\end{array}$ & $0.11 \mathrm{~g} \pm 0.032$ \\
\hline
\end{tabular}

embryo implantation after open surgery. (f) Representative images of embryos (day 16) obtained from BoTA-treated or saline-treated mouse uterus. (g-h) Average weight of embryos obtained from BoTA-treated uterus compared to control. Data shown for (d) and (g) are from 3 independent experiments (\# of mice, 17; \# of embryos, 157) and analyzed using the paired Student $t$-test for parametric distributions including $P$ values $(*<0.05, * *<0.01, * * *<0.001, N S$ not significant).

consistent to the previous findings from reconstructive and aesthetic surgery fields evaluating the angiogenic effect of microenvironment for the embryo implantation. This is 
BoTA application demonstrating increased endothelial cell sprouting with higher numbers of vessel formation in vitro [26] and elevated transverse rectus abdominis myocutaneous flap survival by BoTA-induced angiogenic effect via increased HIF $1 \alpha /$ VEGF signaling [25]. Significant induction of endometrial receptivity-related genes both in cultured endometrial epithelial or stromal cells with BoTA treatment indicates that BoTA induces the expression of those genes even in the independent and separate culture condition of endometrial epithelium or stroma (Fig. 2). Moreover, the positive regulation of BoTA application in the endometrial angiogenesis and receptivity was strongly evidenced by the comparison of RNA-seq of BoTA-treated mouse uterus tissues to salinetreated controls. Thirteen significantly differentially expressed genes, including CCL7, CYR61, ITGB3, FOXC1, HIF3A, GPX1, CD34, CCBE1, TGFBI, LIF, STC1, and STC2, were sorted for $\mathrm{GO}$ terms of angiogenesis or embryo implantation. It has been reported that CCL7, CYR61, FOXC1, and CD34 play crucial roles in vascular remodeling and endothelial cell proliferation [41-44]. Additionally, LIF is known to be strongly related to both angiogenesis and embryo implantation via activation of JAK/STAT and MAPK signaling [45]. STC1 and STC2 have been suggested as key players displaying increased expression levels at the sites of embryo implantation [46]. This has been dramatically supported by the data showing increased levels of CD31 and CD34 expression in intrauterine BoTA-treated mouse endometrial lining compared to control; effects of BoTA in vessel formation were assessed by measuring the total numbers of staining and quantification of stained surface area (Fig. 4b-f). Furthermore, the pregnancy outcomes revealing significantly higher rates of embryo implantation in BoTA-treated horn of mouse uterus compared to the rates in saline-treated uterine horn support RNA-seq analyses (Figs. 4 and 6). However, high concentration of BoTA application restores or even reduces the levels of angiogenesis and endometrial receptivity-related gene expression back to the levels observed in control groups (Supplementary Fig. 1 ), which is consistent to the previous findings showing the interference of angiogenesis by high concentrations of BoTA treatment in endothelial cells in vitro [26]. This might implicate that the dose selection and adjustment might be a critical process for the clinical trial to patients with endometrial problems.

The effect of BoTA on the microvascular remodeling and its activation has been studied to a lesser extent. Consistent with the previous reports demonstrating a significant increase in resting diameter of arterioles and venules with the topical application of BoTA to the rat cremaster muscle [24, 47], in the present study, we have demonstrated that non-invasive intrauterine BoTA administration increases the numbers of microvessel formation in the lining of the endometrium, which has been shown with the assessment of CD31 and CD34 immunoreactivity in the longitudinally sectioned tissues (Fig. 4), and more abundant and thicker uterine arteries were observed surrounding the sites of embryo implantation in the BoTA-applied mouse uterus compared to controls (Fig. $6 \mathrm{~g}$ ). Even though the detailed mechanisms of these actions are still required to be elucidated in the future study, these data might imply that absorbed BoTA from the endometrial surface by the non-invasive intrauterine administration stimulates the formation of neovessels or elongation of existing microvessels towards the innermost lining of the endometrium and also induces the formation of uterine arteries outside the uterus probably to provide better and more fertile environment to the sites of embryo implantation.

A variety of studies evaluated the impact of BoTA on temporary muscular paralysis and relief of the tension on the sites of wound, which may aid to prevent the hypertrophy and hyperpigmentation of wound sites [48, 49]. BoTA is also widely investigated and applied in pain management of myofascial syndrome, headaches, arthritis, and neuropathic pain by induction of muscle chemodenervation via preventing the release of neurotransmitters such as acetylcholine and noradrenaline [50, 51]. A broad range of all these BoTA utilization may affirm its safety in human body, which has been addressed in the previous studies by demonstrating the remarkably effective and safe results of BoTA treatment for the facial aesthetic purposes, obtained from 1474 subjects [52], and quantitatively safe and tolerable profiles of BoTA utilization from the meta-analysis involving 2309 subjects [53]. Consistent with these reports, in our current study, we revealed that BoTA induces neither cytotoxicity, aberrant cell proliferation, nor irregular migratory ability (Supplementary Fig. 2). Moreover, there was no significant difference observed in the weight of day 16 embryos between BoTAtreated and saline-treated groups, especially no retarded embryos were observed in any groups (Fig. 6f-h), suggesting non-invasive intrauterine administration of BoTA as an effective therapeutic strategy with the safety.

Significantly, we demonstrated that increased numbers of microvessels were formed in the mouse endometrium with intrauterine BoTA treatment accompanied with elevated levels of angiogenesis-related marker expression. Additionally, enhanced rates of embryo implantation without any retarded embryos were observed in BoTA-treated mice compared to control group. Taken together, we provided a novel evidence suggesting an intrauterine BoTA administration as an effective therapeutic intervention to improve the endometrial microenvironment by increasing endometrial blood supply. Further study will be required to uncover the molecular mechanisms underlying the induction of angiogenesis by BoTA and direct or indirect association between BoTA-induced endometrial angiogenesis and the embryo implantation. Moreover, it will be essential to evaluate the reproductive toxicity of BoTA intrauterine application prior to clinical trials. A better understanding of uterine endometrial 
angiogenesis induced by BoTA may aid to develop a noninvasive novel method for clinical treatment of repeated implantation failure patients with less risk.

Supplementary Information The online version contains supplementary material available at https://doi.org/10.1007/s43032-021-00496-4.

Acknowledgements We would like to express our sincere gratitude to Dr. Haeng-Seok Song for providing CRL4003 cell line and Dr. Tae Hwan Park for his critical and insightful discussion.

Code Availability Not applicable.

Author Contribution YJK and HSK planned the studies and conducted analysis and interpretation of all experiments; MJY, SHH, and JHA conducted experiments; HJC and DBL performed RNA-seq analyses; CWP contributed to critical discussion; and HSK, JHA, and YJK wrote the manuscript.

Funding This work is supported by funding from the National Research Foundation of Korea (NRF) grant funded by the Korea government (MSIT) to YJK (No. 2018R1C1B6003) and to HSK (No. 2020R1C1C100787211).

Data Availability RNA-seq data that support the findings of this study have been deposited in GEO with the primary accession code GSE146934. The authors declare that all other data supporting the findings of this study are available within the article and its Supplementary information files.

\section{Declarations}

Ethics Approval and Consent to Participate This experimental procedure and animal use protocol have been approved by the Animal Ethics Committee of the CHA University.

Consent to Participate Not applicable.

Consent for Publication Not applicable.

Conflict of Interest The authors declare no competing interests.

Open Access This article is licensed under a Creative Commons Attribution 4.0 International License, which permits use, sharing, adaptation, distribution and reproduction in any medium or format, as long as you give appropriate credit to the original author(s) and the source, provide a link to the Creative Commons licence, and indicate if changes were made. The images or other third party material in this article are included in the article's Creative Commons licence, unless indicated otherwise in a credit line to the material. If material is not included in the article's Creative Commons licence and your intended use is not permitted by statutory regulation or exceeds the permitted use, you will need to obtain permission directly from the copyright holder. To view a copy of this licence, visit http://creativecommons.org/licenses/by/4.0/.

\section{References}

1. Smith SK. Regulation of angiogenesis in the endometrium. Trends Endocrinol Metab. 2001;12(4):147-51. https://doi.org/10.1016/ s1043-2760(01)00379-4.

2. Sherer DM, Abulafia O. Angiogenesis during implantation, and placental and early embryonic development. Placenta. 2001;22(1): 1-13. https://doi.org/10.1053/plac.2000.0588.

3. Gong X, Tong Q, Chen Z, Zhang Y, Xu C, Jin Z. Microvascular density and vascular endothelial growth factor and osteopontin expression during the implantation window in a controlled ovarian hyperstimulation rat model. Exp Ther Med. 2015;9(3):773-9. https://doi.org/10.3892/etm.2015.2181.

4. Geva E, Jaffe RB. Role of angiopoietins in reproductive tract angiogenesis. Obstet Gynecol Surv. 2000;55(8):511-9. https://doi. org/10.1097/00006254-200008000-00024.

5. Jaffe RB. Importance of angiogenesis in reproductive physiology. Semin Perinatol. 2000;24(1):79-81. https://doi.org/10.1016/s01460005(00)80062-2.

6. Smith SK. Angiogenesis, vascular endothelial growth factor and the endometrium. Hum Reprod Update. 1998;4(5):509-19. https://doi. org/10.1093/humupd/4.5.509.

7. Maas JW, Groothuis PG, Dunselman GA, de Goeij AF, Struyker Boudier HA, Evers JL. Endometrial angiogenesis throughout the human menstrual cycle. Hum Reprod. 2001;16(8):1557-61. https:// doi.org/10.1093/humrep/16.8.1557.

8. Dey SK, Lim H, Das SK, Reese J, Paria BC, Daikoku T, et al. Molecular cues to implantation. Endocr Rev. 2004;25(3):341-73. https://doi.org/10.1210/er.2003-0020.

9. Psychoyos A. Hormonal control of ovoimplantation. Vitam Horm. 1973;31:201-56. https://doi.org/10.1016/s0083-6729(08)60999-1.

10. Tabibzadeh S. Molecular control of the implantation window. Hum Reprod Update. 1998;4(5):465-71. https://doi.org/10.1093/ humupd/4.5.465.

11. Chen X, Man GCW, Liu Y, Wu F, Huang J, Li TC, et al. Physiological and pathological angiogenesis in endometrium at the time of embryo implantation. Am J Reprod Immunol. 2017;78(2). https://doi.org/10.1111/aji.12693.

12. Rogers PA. Structure and function of endometrial blood vessels. Hum Reprod Update. 1996;2(1):57-62. https://doi.org/10.1093/ humupd/2.1.57.

13. Kim H, Shin JE, Koo HS, Kwon H, Choi DH, Kim JH. Effect of autologous platelet-rich plasma treatment on refractory thin endometrium during the frozen embryo transfer cycle: a pilot study. Front Endocrinol (Lausanne). 2019;10:61. https://doi.org/10.3389/ fendo.2019.00061.

14. Xie Y, Zhang T, Tian Z, Zhang J, Wang W, Zhang H, et al. Efficacy of intrauterine perfusion of granulocyte colony-stimulating factor (G-CSF) for infertile women with thin endometrium: a systematic review and meta-analysis. Am J Reprod Immunol. 2017;78(2). https://doi.org/10.1111/aji.12701.

15. Zhu X, Peault B, Yan G, Sun H, Hu Y, Ding L. Stem cells and endometrial regeneration: from basic research to clinical trial. Curr Stem Cell Res Ther. 2019;14(4):293-304. https://doi.org/10.2174/ $1574888 X 14666181205120110$.

16. Zhao J, Xu B, Xie S, Zhang Q, Li YP. Whether G-CSF administration has beneficial effect on the outcome after assisted reproductive technology? A systematic review and meta-analysis. Reprod Biol Endocrinol. 2016;14(1):62. https://doi.org/10.1186/s12958016-0197-2. 
17. Du J, Lu H, Yu X, Lu Z, Mi L, Zhang X. Efficacy and safety of platelet-rich plasma for the treatment of thin endometrium: a protocol for systematic review and meta-analysis. Medicine (Baltimore). 2020;99(3):e18848. https://doi.org/10.1097/MD. 0000000000018848 .

18. Kwon KH, Shin KS, Yeon SH, Kwon DG. Application of botulinum toxin in maxillofacial field: Part III. Ancillary treatment for maxillofacial surgery and summary. Maxillofac Plast Reconstr Surg. 2019;41(1):45. https://doi.org/10.1186/s40902-019-0226-0.

19. Sundaram H, Liew S, Signorini M, Vieira Braz A, Fagien S, Swift A, et al. Global Aesthetics Consensus: hyaluronic acid fillers and botulinum toxin type A-recommendations for combined treatment and optimizing outcomes in diverse patient populations. Plast Reconstr Surg. 2016;137(5):1410-23. https://doi.org/10.1097/ PRS.0000000000002119.

20. Chen S. Clinical uses of botulinum neurotoxins: current indications, limitations and future developments. Toxins (Basel). 2012;4(10): 913-39. https://doi.org/10.3390/toxins4100913.

21. Doft MA, Hardy KL, Ascherman JA. Treatment of hyperhidrosis with botulinum toxin. Aesthet Surg J. 2012;32(2):238-44. https:// doi.org/10.1177/1090820X11434506.

22. Escher CM, Paracka L, Dressler D, Kollewe K. Botulinum toxin in the management of chronic migraine: clinical evidence and experience. Ther Adv Neurol Disord. 2017;10(2):127-35. https://doi.org/ $10.1177 / 1756285616677005$.

23. Hsieh PF, Chiu HC, Chen $\mathrm{KC}$, Chang $\mathrm{CH}$, Chou EC. Botulinum toxin A for the treatment of overactive bladder. Toxins (Basel). 2016;8(3). https://doi.org/10.3390/toxins8030059.

24. Aru RG, Songcharoen SJ, Seals SR, Arnold PB, Hester RL. Microcirculatory effects of botulinum toxin A in the rat: acute and chronic vasodilation. Ann Plast Surg. 2017;79(1):82-5. https://doi.org/10.1097/SAP.0000000000001054.

25. Park TH, Lee SH, Park YJ, Lee YS, Rah DK, Kim SY. Presurgical botulinum toxin A treatment increases angiogenesis by hypoxiainducible factor-1alpha/vascular endothelial growth factor and subsequent superiorly based transverse rectus abdominis myocutaneous flap survival in a rat model. Ann Plast Surg. 2016;76(6):723-8. https://doi.org/10.1097/SAP. 0000000000000435 .

26. Gugerell A, Kober J, Schmid M, Buchberger E, Kamolz LP, Keck M. Botulinum toxin A: dose-dependent effect on reepithelialization and angiogenesis. Plast Reconstr Surg Glob Open. 2016;4(8):e837. https://doi.org/10.1097/GOX.0000000000000852.

27. Kang YJ, Lees M, Matthews LC, Kimber SJ, Forbes K, Aplin JD. MiR-145 suppresses embryo-epithelial juxtacrine communication at implantation by modulating maternal IGF1R. J Cell Sci. 2015;128(4):804-14. https://doi.org/10.1242/jcs.164004.

28. Chung M, Ahn J, Son K, Kim S, Jeon NL. Biomimetic model of tumor microenvironment on microfluidic platform. Adv Healthc Mater. 2017;6(15). https://doi.org/10.1002/adhm.201700196.

29. Kang YJ, Forbes K, Carver J, Aplin JD. The role of the osteopontinintegrin alphavbeta 3 interaction at implantation: functional analysis using three different in vitro models. Hum Reprod. 2014;29(4): 739-49. https://doi.org/10.1093/humrep/det433.

30. Carver J, Martin K, Spyropoulou I, Barlow D, Sargent I, Mardon H. An in-vitro model for stromal invasion during implantation of the human blastocyst. Hum Reprod. 2003;18(2):283-90. https://doi. org/10.1093/humrep/deg072.

31. Zhou $\mathrm{X}, \mathrm{Xu} \mathrm{B}$, Zhang $\mathrm{D}$, Jiang $\mathrm{X}$, Chang HM, Leung PCK, et al. Loss of CDYL Results in suppression of ctnnb1 and decreased endometrial receptivity. Front Cell Dev Biol. 2020;8:105. https:// doi.org/10.3389/fcell.2020.00105.
32. Mlecnik B, Galon J, Bindea G. Automated exploration of gene ontology term and pathway networks with ClueGO-REST. Bioinformatics. 2019;35(19):3864-6. https://doi.org/10.1093/ bioinformatics/btz163.

33. Huang da W, Sherman BT, Lempicki RA. Systematic and integrative analysis of large gene lists using DAVID bioinformatics resources. Nat Protoc. 2009;4(1):44-57. https://doi.org/10.1038/ nprot.2008.211.

34. Shin M, Beane TJ, Quillien A, Male I, Zhu LJ, Lawson ND. Vegfa signals through ERK to promote angiogenesis, but not artery differentiation. Development. 2016;143(20):3796-805. https://doi. org/10.1242/dev.137919.

35. Zhang EY, Gao B, Shi HL, Huang LF, Yang L, Wu XJ, et al. 20(S)Protopanaxadiol enhances angiogenesis via HIF-1alpha-mediated VEGF secretion by activating p70S6 kinase and benefits wound healing in genetically diabetic mice. Exp Mol Med. 2017;49(10): e387. https://doi.org/10.1038/emm.2017.151.

36. Aplin JD, Ruane PT. Embryo-epithelium interactions during implantation at a glance. J Cell Sci. 2017;130(1):15-22. https://doi. org/10.1242/jcs. 175943.

37. Siemerink MJ, Klaassen I, Vogels IM, Griffioen AW, Van Noorden CJ, Schlingemann RO. CD34 marks angiogenic tip cells in human vascular endothelial cell cultures. Angiogenesis. 2012;15(1):15163. https://doi.org/10.1007/s10456-011-9251-z.

38. Rizov M, Andreeva P, Dimova I. Molecular regulation and role of angiogenesis in reproduction. Taiwan J Obstet Gynecol. 2017;56(2):127-32. https://doi.org/10.1016/j.tjog.2016.06.019.

39. Park DW, Yang KM. Hormonal regulation of uterine chemokines and immune cells. Clin Exp Reprod Med. 2011;38(4):179-85. https://doi.org/10.5653/cerm.2011.38.4.179.

40. Koo HS, Park CW, Cha SH, Yang KM. Serial evaluation of endometrial blood flow for prediction of pregnancy outcomes in patients who underwent controlled ovarian hyperstimulation and in vitro fertilization and embryo transfer. J Ultrasound Med. 2018;37(4): 851-7. https://doi.org/10.1002/jum.14418.

41. Babic AM, Kireeva ML, Kolesnikova TV, Lau LF. CYR61, a product of a growth factor-inducible immediate early gene, promotes angiogenesis and tumor growth. Proc Natl Acad Sci U S A. 1998;95(11):6355-60. https://doi.org/10.1073/pnas.95.11.6355.

42. Hashimoto T, Shibasaki F. Hypoxia-inducible factor as an angiogenic master switch. Front Pediatr. 2015;3:33. https://doi.org/10. 3389/fped.2015.00033.

43. Popa ER, Harmsen MC, Tio RA, van der Strate BW, Brouwer LA, Schipper M, et al. Circulating CD34+ progenitor cells modulate host angiogenesis and inflammation in vivo. J Mol Cell Cardiol. 2006;41(1):86-96. https://doi.org/10.1016/j.yjmcc.2006.04.021.

44. Qi D, Wei M, Jiao S, Song Y, Wang X, Xie G, et al. Hypoxia inducible factor 1alpha in vascular smooth muscle cells promotes angiotensin II-induced vascular remodeling via activation of CCL7mediated macrophage recruitment. Cell Death Dis. 2019;10(8):544. https://doi.org/10.1038/s41419-019-1757-0.

45. Suman P, Malhotra SS, Gupta SK. LIF-STAT signaling and trophoblast biology. JAKSTAT. 2013;2(4):e25155. https://doi.org/10. $4161 /$ jkst.25155.

46. Xiao LJ, Yuan JX, Song XX, Li YC, Hu ZY, Liu YX. Expression and regulation of stanniocalcin 1 and 2 in rat uterus during embryo implantation and decidualization. Reproduction. 2006;131(6): 1137-49. https://doi.org/10.1530/rep.1.01100.

47. Stone AV, Koman LA, Callahan MF, Eckman DM, Smith BP, Plate JF, et al. The effect of botulinum neurotoxin-A on blood flow in rats: a potential mechanism for treatment of Raynaud 
phenomenon. J Hand Surg [Am]. 2012;37(4):795-802. https://doi. org/10.1016/j.jhsa.2012.01.021.

48. Al-Qattan MM, Al-Shanawani BN, Alshomer F. Botulinum toxin type A: implications in wound healing, facial cutaneous scarring, and cleft lip repair. Ann Saudi Med. 2013;33(5):482-8. https://doi. org/10.5144/0256-4947.2013.482.

49. Ziade M, Domergue S, Batifol D, Jreige R, Sebbane M, Goudot P, et al. Use of botulinum toxin type A to improve treatment of facial wounds: a prospective randomised study. J Plast Reconstr Aesthet Surg. 2013;66(2):209-14. https://doi.org/10.1016/j.bjps.2012.09.012.

50. Colhado OC, Boeing M, Ortega LB. Botulinum toxin in pain treatment. Rev Bras Anestesiol. 2009;59(3):366-81. https://doi.org/10. 1590/s0034-70942009000300013.
51. Sim WS. Application of botulinum toxin in pain management. Korean J Pain. 2011;24(1):1-6. https://doi.org/10.3344/kjp.2011.24.1.1.

52. Guo Y, Lu Y, Liu T, Zhou Y, Yang P, Zhu J, et al. Efficacy and safety of botulinum toxin type $\mathrm{A}$ in the treatment of glabellar lines: a meta-analysis of randomized, placebo-controlled, double-blind trials. Plast Reconstr Surg. 2015;136(3):310e-8e. https://doi.org/ 10.1097/PRS.0000000000001544.

53. Naumann M, Jankovic J. Safety of botulinum toxin type A: a systematic review and meta-analysis. Curr Med Res Opin. 2004;20(7): 981-90. https://doi.org/10.1185/030079904125003962.

Publisher's Note Springer Nature remains neutral with regard to jurisdictional claims in published maps and institutional affiliations. 